\title{
Changes in articular cartilage in experimentally induced patellar subluxation
}

Department of Orthopaedic Surgery, Nihon University School of Medicine, Tokyo, Japan

J Ryu

S Saito

K Yamamoto

Correspondence to: Dr J Ryu, Department of Orthopaedic Surgery, Nihon University School of Medicine, 30-1 Oyaguch Kamimachi, Itabashiku, Tokyo, Japan 173.

Accepted for publication 1 August 1997

Junnosuke Ryu, Shu Saito, Kazuki Yamamoto

\begin{abstract}
Objectives-Patellar subluxation was experimentally induced in young rabbits and the resulting cartilaginous changes were observed over a prolonged period of time to determine histological changes in the subluxated patellar cartilage.

Methods-The tibial tuberosity in 12 week old rabbits was laterally displaced and fixed to the tibia with wire to induce lateral patellar subluxation. Pathological changes in patellar cartilage were examined for 120 weeks after surgery using computed tomography and stereoscopic microscopy.

Results-Eight weeks after surgery, changes in articular cartilage consisting of horizontal splitting of the matrix were observed in the intermediate zone and were presumed to have been caused by shearing stress applied to the patellar cartilage. The cartilaginous changes caused by patellar subluxation progressed very little over the 120 weeks. Very few rabbits presented with osteoarthritic changes in the patellofemoral joint, most probably because the stress resulting from the malalignment of the patellofemoral joint was mild enough to permit recovery. Conclusion-The mild, non-progressive pathological changes, in particular, basal degeneration, induced in this experiment in patellar cartilage were quite similar to the changes in articular cartilage seen in human chondromalacia patellae.
\end{abstract}

(Ann Rheum Dis 1997;56:677-681)

The term chondromalacia patellae is widely used to describe clinical symptoms that include anterior knee pain, unstable feeling in the knee, tenderness on the patella, and giving way, with softening or fibrillation of the articular cartilage of the patella. Many studies have investigated the aetiology, macroscopic pathology, arthroscopic findings, clinical symptoms, and treatment of chondromalacia patellae, but surprisingly few have described the microscopic pathology of the articular cartilage in chondromalacia patellae..$^{1-3}$

The aetiology of chondromalacia has been thought by many to be trauma related. ${ }^{4}$ Abnormal patellofemoral tracking has been reported as an important aetiological factor in the development of chondromalacia patellae. Heywood ${ }^{5}$ showed that patellar dislocation led to chondromalacia patellae in his operated patients. Dandy ${ }^{6}$ operated on 57 knees with chondroma- lacia and unstable patellae and found that chondromalacia patellae was more commonly associated with patellar subluxation (97\%) than with patellar dislocation (62\%). Imai et al ${ }^{7}$ stated that the pathological features of patellar dislocation and subluxation do not differ essentially from those of chondromalacia patellae, and that these names are often used interchangeably. Consequently, this condition is referred to as recurrent dislocation or subluxation when viewed in terms of patellar movement, and chondromalacia patellae when viewed in term of cartilage change.

Karlson ${ }^{8}$ followed up a group of patients with chondromalacia patellae for 20 years and found little evidence of degenerative change in the patellofemoral joint. Furthermore, older patients with osteoarthritis of the patellofemoral joint rarely have a history of chondromalacia patella in adolescence or early adult life. Bentley $^{9}$ stated that the clinical symptoms are self limiting and that chondromalacia patellae does not progress to osteoarthritis in later life, which suggests that the two diseases are different entities.

Previously, we reported early changes in experimentally induced patellar subluxation in rabbits. ${ }^{10}$ In this study, to elucidate the histological changes and condition of the patellar articular cartilage in subluxation of the patellofemoral joint, patellar subluxation, and abnormal shearing forces were induced in the patellar articulation in rabbits by lateral displacement of the tibial tuberosity. Changes in patella articulation were then histologically determined for 120 weeks.

\section{Methods}

INDUCEMENT OF PATELLAR SUBLUXATION

The experiment was performed using $52 \quad 12$ week old female rabbits, each weighing 1500$2500 \mathrm{~g}$. After induction of general anaesthesia using pentobarbitone (sodium pentobarbital) inhalation, a $3 \mathrm{~cm}$ long midline longitudinal incision was made over the right patella, the upper margin of the tibial tuberosity was osteotomised, and the tuberosity freed from the joint. The medial retinaculum was then severed without injuring the articular capsule, and the freed piece of bone was reattached at a position $5 \mathrm{~mm}$ lateral to the original position using 1.0 $\mathrm{mm}$ diameter Kirschner wire. As controls, sham operations were performed on the left patella in the same 52 rabbits. In the sham operation, the same incision performed on the operative knee was also performed on the contralateral patellae, and medial retinacular release without a capsular incision was 
performed. After lateral patellar subluxation was established, the rabbits were returned to their cages and were free to move without any external immobilisation.

EXAMINATION OF THE PATELLAR CARTILAGE

Preparation of specimens

After the inducement of subluxation, four rabbits were killed by a lethal dose of pentobarbitone inhalation at each of $2,4,8,12,16,20,24$, $28,32,60,100$, and 120 weeks and the following analyses of the patella were performed.

Computed tomography (CT) was performed to examine the patellofemoral joints and the degree of subluxation.At the start of this experimental study, the femoral and tibial epiphyseal lines were noted in the radiographic examinations of these young rabbits. However, in 18 of 20 rabbits $(90 \%)$ followed up for 32 to 120 weeks after surgery, the epiphyseal line had already closed by 32 weeks after surgery. The epiphyseal line had disappeared in all the rabbits examined by the end of the experiment (120 weeks).

Macroscopic technique-Both patellae were excised and examined under a stereoscopic microscope (Wild Photo Microscope, M 400) to observe the superficial and transectional surfaces of the patellar cartilage. The average size of the rabbit patellae in this experiment was $10 \mathrm{~mm} \times 10 \mathrm{~mm} \times 5 \mathrm{~mm}$.

Pathological examination-The patellae were excised, fixed in $10 \%$ formalin for 48 hours at $22^{\circ} \mathrm{C}$, and then decalcified with EDTA $(\mathrm{pH}$ 7.2 ) for 72 hours. To minimise the introduction of any technical or procedural differences during section preparation, both experimental and control patellae were embedded in the same paraffin wax block and cut into serial sections. The specimens obtained at the designated periods were stained with haematoxylin and eosin (HE), Safranin-O, alucian blue, and periodic acid Schiff ( PAS ) stains.

Changes in chondrocyte morphology and matrix were classified using a modified version of the histological-histochemical grading system for osteoarthritis described by Mankin et $a l^{11}$
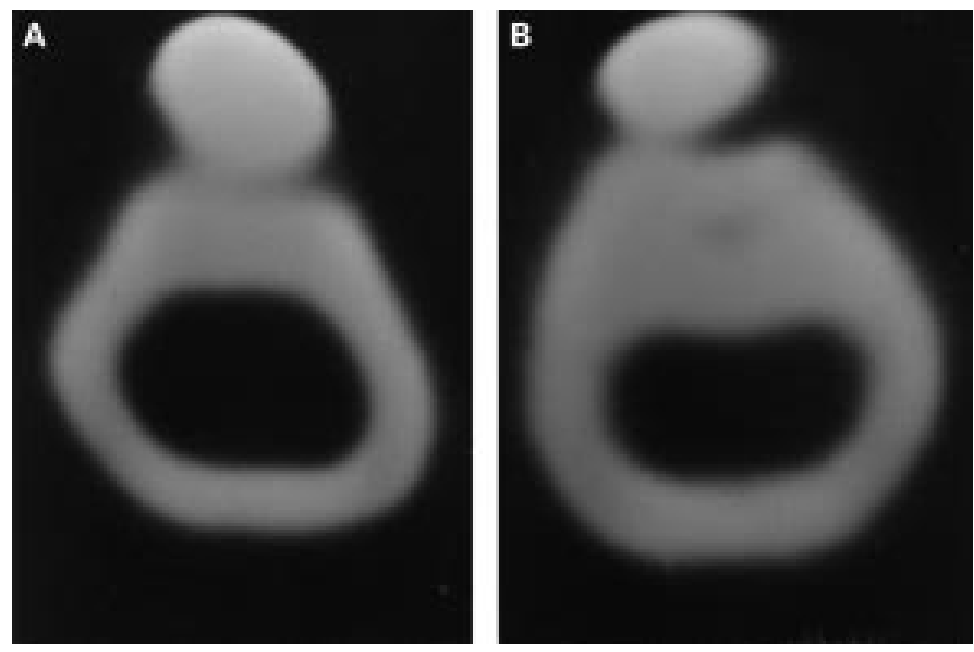

Figure 1 Postoperative CT findings. (A) Control. (B) Lateral shifting of patella and narrowing of lateral joint space in subluxation group.

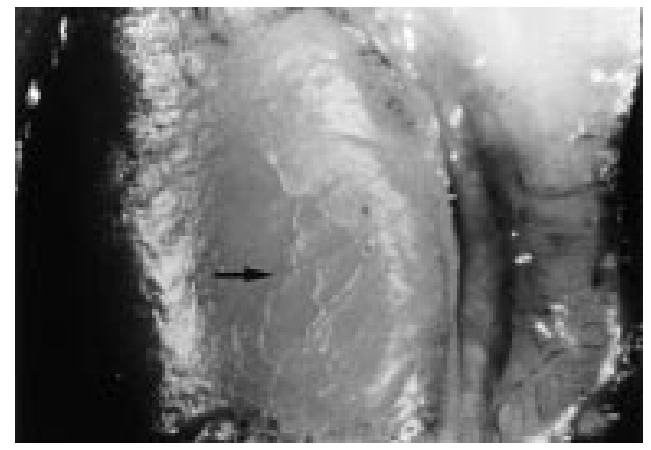

Figure 2 Macroscopic findings in patellar cartilage at eight weeks. Softening, swelling, and irregularity (arrows) are noted on surface of patellar cartilage.

STATISTICAL ANALYSIS

The unpaired $t$ test was used to analyse the incidence of histological change in articular cartilage. Significance was established at the $\mathrm{p}<0.05$ level.

\section{Results}

COMPUTED TOMOGRAPHY

In 46 rabbits $(88.5 \%)$, CT showed lateral deviation of the right patella and narrowing of the lateral joint space of the patellofemoral joint compared with the controls (fig 1). Over the course of this study, CT examination showed that total lateral dislocation of the patella occurred in six $(11.5 \%)$ of 52 rabbits at 24 weeks; these rabbits were excluded from the study and six replacements were prepared and used. No patellar subluxation was seen in any of the control patellae.

\section{MACROSCOPIC FINDINGS}

Changes in patellar cartilage

At surgery, the magnitude of the displacement of the patella was $2-3 \mathrm{~mm}$ in each joint. Four weeks after surgery, the cartilaginous surface of the proximal lateral portion of the patella was observed to have lost some luster in all experimental patellae.

At eight weeks, the cartilaginous surface in all experimental patellae showed malacia and swelling as well as irregular contours (fig 2).

At 24 and 32 weeks, the superficial changes in the patellar cartilage showed no change from those noted at eight weeks, and consisted of softening, swelling, and macroscopic irregularities. These superficial lesions were, on the whole, localised to the proximal lateral facet and the central ridge of the patellae. No changes were noted in the medial facet. No changes were noted in the patellar cartilage of the control joints.

HISTOLOGICAL FINDINGS

Changes in synovial membrane

Up to 20 weeks after the inducement of subluxation, upon observation of the synovial membrane around the affected patellae, slight or moderate chronic synovitis such as hyperplasia of the synovial membrane with lymphocyte and mononuclear cell infiltration, vasculitis of the small vessels in the stroma, and proliferation of lining cells was detected microscopically around the affected patella. However, thereafter, the 
Table 1 Incidence of horizontal splits in relation to change in chondrocytes and matrix

\begin{tabular}{|c|c|c|c|c|c|}
\hline \multirow[b]{2}{*}{ Weeks } & \multirow{2}{*}{$\begin{array}{l}\text { Safranin-0 } \\
\text { staining }\end{array}$} & \multicolumn{2}{|c|}{$\begin{array}{l}\text { Incidence of horizontal } \\
\text { splits }\end{array}$} & \multirow[b]{2}{*}{ Size of splits } & \multirow[b]{2}{*}{ Changes in chondrocytes } \\
\hline & & Present & Absent & & \\
\hline 2 & $0 \times 4$ & 0 & 4 & 0 & 0 \\
\hline 4 & $0 \times 2,1 \times 2$ & 0 & 4 & 0 & 0 \\
\hline 8 & $0 \times 1,1 \times 3$ & 2 & 2 & $1 \times 2$ & 1 \\
\hline 12 & $1 \times 1,2 \times 3$ & 2 & 2 & $2 \times 1,3 \times 1$ & 1 \\
\hline $16^{\star}$ & $2 \times 4$ & 3 & 1 & $1 \times 1,2 \times 2$ & 1 \\
\hline $20^{\star}$ & $2 \times 3,3 \times 1$ & 3 & 1 & $2 \times 2,3 \times 1$ & 1 \\
\hline $24^{\star}$ & $2 \times 3,3 \times 1$ & 3 & 1 & $2 \times 2,3 \times 1$ & 1 \\
\hline $28^{\star}$ & $2 \times 2,3 \times 2$ & 2 & 2 & $3 \times 2$ & 1 \\
\hline 32 & $2 \times 3,3 \times 1$ & 2 & 2 & $1 \times 2$ & 1 \\
\hline 60 & $2 \times 2,3 \times 2$ & 1 & 3 & $1 \times 1$ & 1 \\
\hline 80 & $1 \times 3,2 \times 1$ & 0 & 4 & 0 & 0 \\
\hline 100 & $1 \times 4$ & 0 & 4 & 0 & 0 \\
\hline 120 & $1 \times 4$ & 0 & 4 & 0 & 0 \\
\hline \multicolumn{6}{|c|}{$\begin{array}{l}\text { Changes in chondrocytes and safranin- } 0 \text { staining of matrix were graded using a modification of } \\
\text { the grading system defined by Mankin et al. }{ }^{11}\end{array}$} \\
\hline \multicolumn{4}{|c|}{ Chondrocytes } & & Grade \\
\hline \multicolumn{4}{|c|}{ Normal } & & 0 \\
\hline \multicolumn{4}{|c|}{ Diffuse hypercellularity } & & 1 \\
\hline \multicolumn{4}{|c|}{ Cloning } & & 2 \\
\hline \multicolumn{4}{|c|}{ Hypocellularity } & & 3 \\
\hline \multicolumn{6}{|c|}{ Safranin-0 staining } \\
\hline \multicolumn{4}{|c|}{ Normal } & & 0 \\
\hline \multicolumn{4}{|c|}{ Slight reduction } & & 1 \\
\hline \multicolumn{4}{|c|}{ Moderate reduction } & & 2 \\
\hline \multicolumn{4}{|c|}{ Severe reduction } & & 3 \\
\hline \multicolumn{4}{|c|}{ No dye noted } & & $\begin{array}{l}4 \\
\left(\text { From Mankin et } a l^{11}\right)\end{array}$ \\
\hline \multicolumn{6}{|c|}{ Size of splits } \\
\hline \multicolumn{4}{|c|}{ None } & & 0 \\
\hline \multicolumn{2}{|c|}{ Minor } & & & & 1 \\
\hline \multirow{2}{*}{\multicolumn{2}{|c|}{$\begin{array}{l}\text { Intermediate } \\
\text { Maior }\end{array}$}} & & & & 2 \\
\hline & & & & & 3 \\
\hline
\end{tabular}

«Statistical comparison of incidence of horizontal splitting $\mathrm{p}<0.001$.

chronic synovitis declined, and, from 32 weeks onward, little change was noted in the peripatellar synovial membrane. No change was noted in the synovial membrane of the joints from 60 to 120 weeks after surgery. No pathological
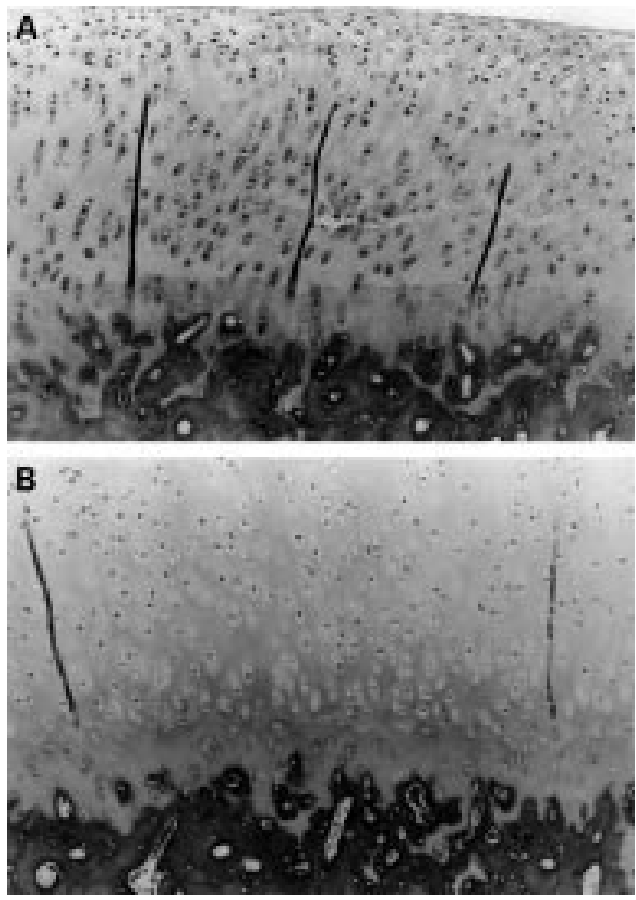

Figure 3 (A) Histological changes in patellar cartilage at eight weeks. Horizontal splits and oedematous changes in matrix in intermediate zones were evident. Surface of cartilage is smooth and no changes in chondrocytes were noted in superficial zone. (Safranin-O, original

magnification $\times 100$ ). (B) Patellar articular cartilage of control patellae at eight weeks. No remarkable change was noted. (Safranin-O, original magnification $\times 120$ ).

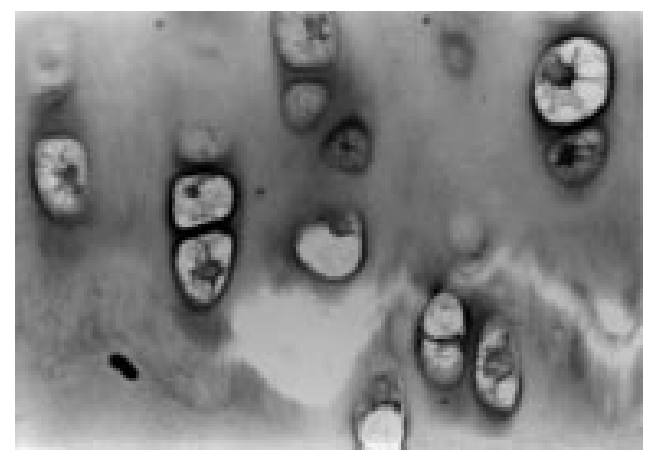

Figure 4 Histological changes around horizontal splits in intermediate zone at eight weeks. In vicinity of splits, chondrocytes of various sizes and with a number of vacuoles in cytoplasm were evident as well as reduction in matrix Safranin-O staining. (Safranin-O, original magnification $\times 200$ ).

changes were seen in the synovial membrane of the control patellae.

Changes in the patellar articular cartilage (table 1) No histological changes were observed two weeks after surgery. However, at four weeks, the cartilage of two rabbits showed a decrease in Safranin-O staining compared with the control patellae.

At eight weeks, horizontal splits (clefts), parallel to the facet, were found in the intermediate zones of the patellar cartilage in two of four rabbits examined. Histochemically, the patellar cartilage showed oedematous changes around the horizontal splits in the middle zones of the cartilage matrix. Furthermore, the proteoglycan
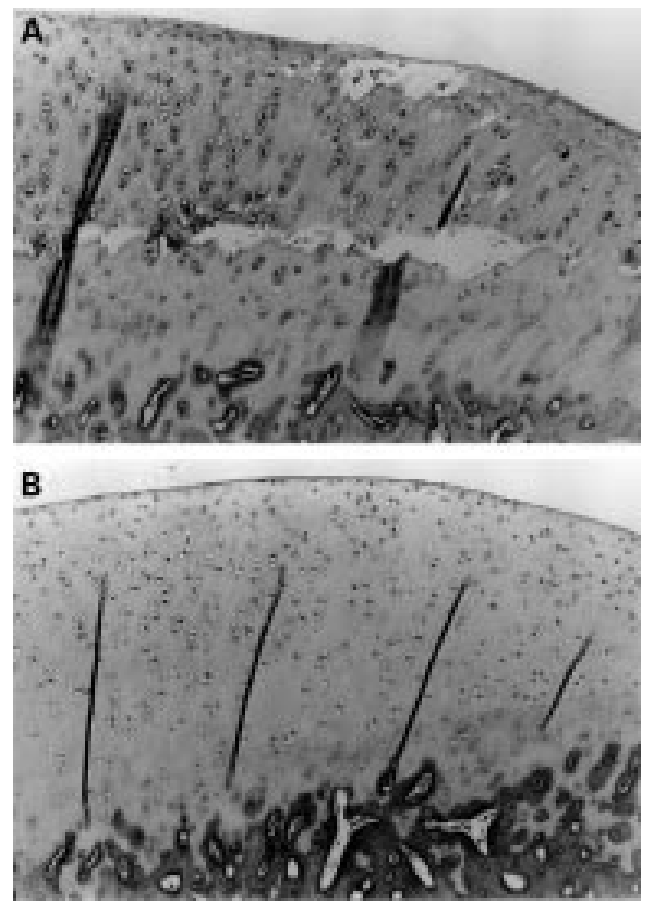

Figure 5 (A) At 12 weeks, horizontal splits have expanded widely and transversely parallel to surface with blister formation on articular surface and reduction in Safranin-O staining around blister is evident. Morphology of chondrocytes in superficial layer and deep zone differs. (Safranin-O, original magnification $\times 100)$. (B) Patellar articular cartilage of control side at 12 weeks. No remarkable change is noted in chondrocytes matrix.

$($ Safranin-O, original magnification $\times 100)$. 

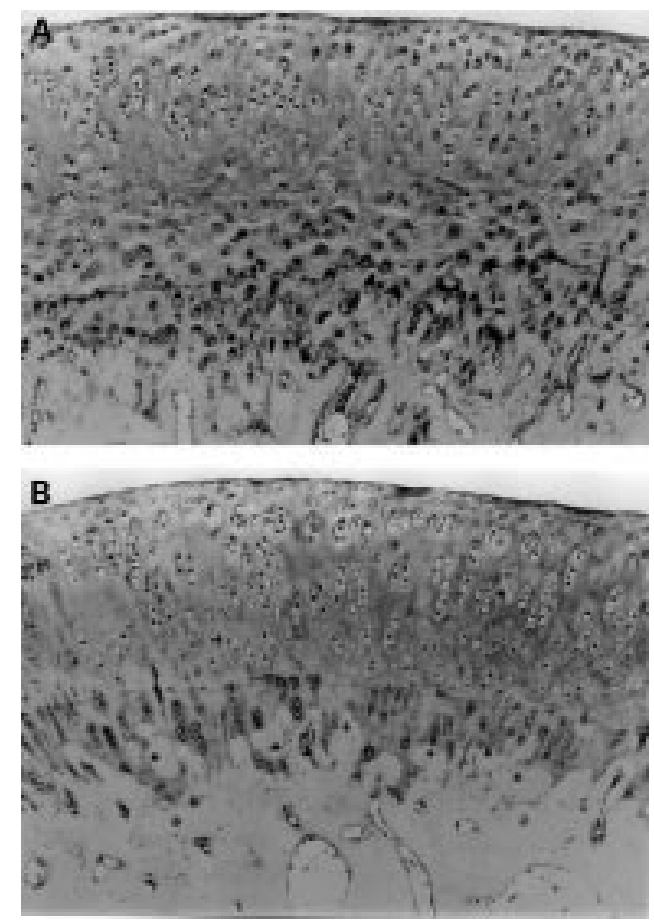

Figure 6 (A) At 32 weeks, cartilage is oedematous and many splits and wrinkles of matrix are evident in intermediate zones of patellar. Chondrocytes of superficial zone are not morphologically different to chondrocytes of control. (Safranin-O, original magnification $\times 100$ ). (B) Patellar cartilage at 32 weeks in control. No remarkable changes noted in chondrocytes and matrix. (Safranin-O, original magnification $\times 100$ ).

concentration in the matrix around the splits had decreased as indicated by the reduction in Safranin O staining of the area. In these patellae, the tide marks had become irregular (fig 3).

At 12 weeks, similar but more extensive horizontal splits, were noted in the same area in two of four rabbits examined. Empty lacunae had developed in the cartilage around the splits (fig 4). In one rabbit, a blister had developed on the cartilagenous surface (fig 5), and in three, the lateral margin of the patellar cartilage was covered with fibrous connective tissue extending from the synovial membrane.

At 24 weeks, extensive horizontal splitting was seen in three of four rabbits and Safranin-O staining was reduced to moderate in one and slight in two.

At 32 weeks, the pathological changes showed very little progression compared with those seen at 12 weeks. The intermediate zones were oedematous, with many small horizontal split stills present (fig 6).

At 60 weeks, irregular cell lines, oedema of stroma, and small multiple horizontal splits in the intermediate zone (one of four) were noted but the other patellae showed no significant progression of the changes seen at 32 weeks.

At 120 weeks, empty lacunae, disappearance of chondrocytes, and irregularity of the tidemarks was observed, but horizontal splits in the intermediate zone seen at 60 weeks were noted. Even after 120 weeks, the superficial lesions showed no signs of progressing toward any condition resembling $\mathrm{OA}$, the histological characteristics of which include fibrillation of the patellar surface, cluster formations of chondrocytes and matrical changes, formation of vertical clefts, and thinning of the cartilagenous layer.

The control patellar cartilage, embedded in the same paraffin wax block and sectioned with the subluxed patellae, was free from histopathological changes, as were the cartilaginous specimens from the patellofemoral joint, which had also been prepared simultaneously with the patellar specimens.

\section{Discussion}

The results of this study of an experimental rabbit model for patellar subluxation showed four, seemingly characteristic, patterns. Firstly, the changes were confined predominantly to the proximal lateral facet of the patellar cartilage, where the shear stress of subluxation was most likely to occur. Secondly, the changes developed in the intermediate to deep zones rather than in the superficial zone. Thirdly, by eight weeks, horizontal splits, parallel to the facet, developed in the intermediate zone in more than half of the patellae examined. Fourthly, these changes progressed so slowly that little difference from those observed at 12 weeks was noted at 120 weeks. Very slight arthritic changes in the synovial membrane developed around the patellofemoral joint.

In this study of experimentally induced patellar subluxation, the changes that developed in the lateral facets of patellar articular cartilage occurred where the shearing pressure was highest. Mollen et $a l,{ }^{12}$ who also examined experimental subluxation of rabbit patellae, also reported that the lesions developed in the central ridge and lateral facets of the patellae, which are the sites most subjected to pressure. In this study, however, the force exerted on the lateral facet semed not to be a simple persistent compressive pressure but a shearing stress concomitant with patellar movements, which was noted by the dynamic alignment of the patellofemoral joint. In a human necropsy study, Meachim and Bentley ${ }^{13}$ found horizontal splits in the lateral segments and odd facets of patellar articular cartilage that were assumed to be secondary to shear stress damage. Imai et $a l^{14}$ clinically, as well as experimentally, demonstrated that disturbance of this alignment caused an increase in shear stress on the cartilage, which eventually led to chondromalacia patellae.

Most of the lesions in this study developed in the intermediate zones, rather than in the superficial zones of the patellar cartilage. Ohno et $a l^{15}$ have observed the cartilaginous lesions of human CMP using an electron microscope and have pointed out that the surface structure is typically preserved in CMP (closed CMP).

Thompson et $a l^{16}$ reported the changes in patellar articular cartilage in experimentally induced tansarticular load by applying the drop tower method to canine patellofemoral joints. The fractures created by the load were seen in the calcified cartilage zone with minimum damage to the articular cartilage surface. Six months after loading, the initial changes had progressed to an osteoarthritic-like condition. Although similar to the Thompson experiment, 
the present study differed in that vertical clefts were not seen and horizontal clefts parallel to the surface were noted in the changes in the patellar cartilage of our experiment, the duration and direction of the pressure loading to the patella femoral joint was not temporary and vertical but repeated and shearing, and the changes in articular cartilage did not progress to osteoarthritic-like changes over 120 weeks.

It may be argued that the horizontal splits seen in this experiment were artificially produced in the course of section preparation. However, this is unlikely because the control patellar specimens did not develop horizontal splits despite being simultaneously prepared and resected with the experimental patellae. In addition, the content of splits were susceptible to staining and the area around the splits showed a reduction in Safranin-O staining. Whether such clefts actually develop in the patellar cartilage of humans is unclear. Meachin and Bentley ${ }^{13}$ have reported that horizontal splitting at the interface between the uncalcified and calcified layers of the articular cartilage was found in 31 of 50 transverse slabs of patellae in a random series of 50 necropsy subjects aged 18 to 96 years. The splits were not caused by autolysis nor by technical artefacts, suggesting that they resulted from shearing damage at the uncalcified-calcified cartilage interface during life. This possibility has also been experimentally demonstrated by Tomatsu et $a l,{ }^{17}$ who, by applying a shear stress to the patellar cartilage of humans, were able to produce various lesions, including horizontal splits parallel to the facet in the deep zone. In this experiment, the changes developed in the intermediate zones, with horizontal splitting parallel to the facet.

In this study, the cartilaginous changes resulting from patella subluxation progressed very slowly over a long period of time and did not show the typical osteoarthritic-like changes in articular cartilage even at 120 weeks. One possible explanation is that the stress caused by the malalignment of the patellofemoral joint was mild enough to allow recovery of damage to the articular cartilage. LaBerge $e t a l^{18}$ examined the patellar cartilaginous response to experimentally induced static compressive loading in closed chondromalacia patellae in canines. They concluded that an increase in cartilage compliance is associated with the intrinsic remodelling of the cartilage matrix, and that these changes might occur without any external signs of degeneration. Bently ${ }^{9}$ reported that if for any reason the trauma to the articular cartilage of the patella is reduced, for example, by reduced activity or by realignment, repair of cartilage may occur and if the damage has been minimal, complete surface repair may result. Another interpretation of the slow and mild damage to patellar cartilage is that the stress on the cartilage was not a compressive but a shear force, which produced damage not to the surface, but rather to the intermediate zone of the cartilage, thus sparing deterioration of the articular cartilage to the point of arthritic changes. In addition, we suggest that the patellofemoral joint has a dynamic alignment during knee flexion and extension. Therefore, subluxated patellofemoral joints gradually adapt to mild shearing forces and the cartilage injury that does develop gradually heals before osteoarthritis develops.

Patellar subluxation was induced in rabbits and the resulting cartilaginous changes were observed over 120 weeks. Pathological changes, primarily horizontal splitting parallel to the facets, developed in the proximal lateral patellar cartilage and most of these changes were localised to the intermediate zone, with the superficial zone being spared. These changes were quite similar to those in human chondromalacia patellae cartilage in terms of the absence of superficial lesions, the presence of basal degeneration, and the slow progression of major cartilaginous findings. During the long observation period, the lesions rarely progressed, and signs indicative of osteoarthritis were rare.

We would like to thank Professor Henry J Mankin, MD, Orthopaedic Service, Massachusetts General Hospital, Boston, for his paedic Service, Massachusetts General Hospition
kind suggestions and revision of the article.

1 Wiles P, Andrews PS, Devas MB. Chondromalacia of the patella. J Bone Joint Surg 1956;38B:95-113.

2 Darracott J, Vernon-Roberts B. The bony changes in chondromalacia patellae. Rheum Phys Med 1971;11:175-9.

3 Goodfelow JW, Hungerford DS, Wood C. Patello-femoral mechanics and pathology. 2. Chondromalacia patellae. J Bone Joint Surg 1976;58-B:291-9.

4 Bandai W, Brenwald J. The significance of femoropatellar pressure in the pathogenesis and treatment of chondromaOS, Van Linge B, Van Rens ThJG, Rösingh GE, Veraart OS, Van Linge B, Van Rens ThJG, Rösingh GE, Veraart BEEMJ, Le Vay D, eds. The knee join
Elesevier Publishing, 1974:63-8.

5 Heywood AWB. Recurrent dislocation of the patella. A study of its pathology and treatment in 106 knees. J Bone study of its pathology and tretr

6 Dandy DJ, Poirier H. Chondromalacia and the unstable patella. Acta Orthop Scand 1975;4:695-9.

7 Imai N, Tomatsu T. Chondromalacia patellae. Tokyo: Tokai University Press, 1995:7-12.

8 Karlson S. Chondromalacia patellae. Acta Chir Scand 1939;83:347-81.

9 Bently G. Articular cartilage changes in chondromalacia patellae. J Bone Joint Surg 1985;67-B:769-74.

10 Saito S, Ryu J, Yamamoto K, Kohno H. An experimental study on early changes of articular cartilages in subluxated patella of rabbits. J Jpn Orthop Assoc 1991;65:571-9 (in Japanese ).

11 Mankin HJ, Dorfman H, Lippiello L, Zarins A. Biochemical and metabolic abnormalities in articular cartilage from osteoarthritic human hip. II Correlation of morphology with biochemical and metabolic data. J Bone Joint Surg 1971;53A:523-37.

12 Mollen BN, Larsen FM, Frich LH. Chondromalacia Mollen BN, Larsen FM, Frich LH. Chondromalacia Scand 1985;60:188-91.

13 Meachim G, Bentley G. Horizontal splitting in patellar articular cartilage. Arthritis Rheum 1987;21:669-74

14 Imai N, Tomatsu T, Okamoto H, Nakamura Y. Clinical and roentgenological studies on malalignment disorders of the patellofemoral joint, part 3. Lesions of the patellar cartilage and subchondral bone associated with patellofemoral malalignment. J Jpn Orthop Ass 1983;63:1-17.

15 Ohno O, Naito J, Iguchi T, Ishikawa H, Hirohata K. An electron microscopic study of early pathology in chondro-
malacia of the patella. J Bone Joint Surg 1988;70A:883-99.

16 Thompson Jr RC, Theodore R, Oegema Jr TR, Lewis JL, Wallace L. Osteoarthrotic changes after acute transarticular load. J Bone Joint Surg 1991;73A:990-1001.

17 Tomatsu T, Imai N, Takeuchi N, Takahashi K, Kimura N. Experimentally produced fracture of cartilage and bone. Experimentally produced fracture of cartilage and bone.
The effect of shear forces on the pig knee. J Bone Joint Surg 1992;74B:557-62.

18 Laberge M, Audet J, Drouin G, Rivard CH. Structural and in vivo mechanical characterization of canine patellar cartilage: a closed chondromalacia patellae model. J Invest Surg 1993;6:105-16. 